\title{
Prediction Algorithm For Mobile Ad Hoc NETWORK CONNECTION BREAKS
}

\author{
Yasir Mohammed ${ }^{1}$, Maha Abdelhaq ${ }^{2}$ and Raed Alsaqour ${ }^{3}$ \\ ${ }^{1}$ IT Center and System, Iraqi Ministry of Electricity, Baghdad, Iraq \\ ${ }^{2}$ Department of Information Technology, College of Computer and Information Sciences, \\ Princess Nourah bint Abdulrahman University, 84428 Riyadh, Saudi Arabia \\ ${ }^{3}$ Department of Information Technology, College of Computing and Informatics, Saudi \\ Electronic University, 93499 Riyadh, Saudi Arabia
}

\begin{abstract}
A Mobile Ad-Hoc Network (MANET) is a decentralized network of mobile node that are connected to an arbitrary topology via wireless connections. The breakdown of the connecting links between adjacent nodes will probably lead to the loss of the transferred data packets. In this research, we proposed an algorithm for link prediction (LP) to enhance the link break provision of the ad hoc on-demand remote protocol (AODV). The proposed algorithm is called the AODV Link Break Prediction (AODVLBP). The AODVLBP prevents link breaks by the use of a predictive measure of the changing signal. The AODVLBP was evaluated using the network simulator version 2.35 (NS2) and compared with the AODV Link prediction $(A O D V L P)$ and the AODV routing protocols. The simulation results reveal the effectiveness of $A O D V L B P$ in improving network performance in terms of average end-to-end delay, packet delivery ratio, packet overhead ratio, and packet drop-neighbour break.
\end{abstract}

\section{KEYWORDS}

MANET, AODV, Link break, Prediction, Routing protocol

\section{INTRODUCTION}

Mobile Ad-Hoc Network (MANET) is a powerfully improved able of remote network with no static framework. Every host stands for as a router and proceeds in a discretionary way. For a live connection, the end host and the transitional nodes can be mobile [1,2]. Thus, routes are inclined to break much of the time, which prompts as often as possible rerouting to discover another connection to repair or a recently accessible way to recouping correspondence [3,4]. The benefits of wireless ad hoc networks are as per the following. They are quickly deployable and proper for the condition were setting up or keeping up a conveying framework is troublesome or infeasible. For instance, calamity recuperation (quake, fire), a war zone, law implementation, and vehicle-tovehicle networking in savvy transportation frameworks. Also, the mobile nodes convey the capacity to travel openly with no pressure [5].

Nevertheless, MANET likewise has drawbacks. The battery life is aptly limited, and the radio recurrence brings about impedance. Above all, the network topology may adjust continually as a result of node activity $[6,7]$. MANET protocols can generally be subdivided into three main classes: first, proactive routing protocols, or table-driven protocols. The second is on-demand or reactive routing protocols and the third is hybrid protocols. 
In proactive or table-driven routing protocols, there are examples such as Destination Sequenced Demand Vector (DSDV) and Optimized Link State Routing Protocols (OLSR) [8]. In these protocols, each node maintains routing information about the available paths in the network even if these paths are not currently used. Each node uses routing lists updated periodically to store the paths position information of other nodes in the network. This information is used for the transfer of data between different network nodes. Proactive protocol routing could not be seen as an efficient MANET routing solution. The high mobility, large router lists, and poor scalability contribute to node bandwidth and battery life consumption. In addition, the maintenance of unused paths may occupy a significant part of the available bandwidth if the topology of the network changes frequently

Contrasted with proactive protocols, a node begins a route discovery all through the network in reactive or on-demand protocols, such as Dynamic Source Routing (DSR) and AODV, just when it requires sending bundles to its destination. This procedure is done when a route is resolved, or every conceivable stage has been evaluated. When a course has been begun, it is continued by a continuation route procedure either until the objective gets to be inaccessible alongside every way from the source or until the route is no more needed. In the reactive protocol, nodes keep the courses to active destinations [9].

Hybrid routing protocols are a combination of proactive and reactive routing protocols and overcome their weaknesses. Generally, hybrid routing protocols in MANET utilize hierarchical network structural design. Appropriate proactive and reactive routing methods are hard-done-by at different hierarchical levels, one-to-one. The Zone Routing Protocol (ZRP) and Zone-based Hierarchical Link State (ZHLS) routing protocol are examples of MANET hybrid routing protocols [10].

The periodically changing in network topology in routing protocols presents several challenges in MANET that affect the performance of the network. One of the greatest problems is the issue of a link failure in a typical MANET; the nodes act as routers. Since that, a failure of one or more links to that node generally means that packets will be dropped and requires retransmission. While a link failure happens, it is necessary to discover a new path to avoid wastage of the scare node resources.

This research is mainly aimed at giving priority to link repair and/or link discovery. In order to estimate the stability of a route consisting of multiple links, it is necessary to assess which links are likely to become unavailable. A very substantial part of the MANET algorithms is devoted to linking management and prediction. The proposed algorithm was developed to estimate when the signal strength of a link will fall below some given threshold. This is used to demonstrate that the two nodes are moving away from each other. The link prediction algorithm acts as a proactive algorithm in that it warns that source that a new path must be found before the existing path has collapsed. This gives the node of origin the opportunity for path discovery while an existing path is still in place.

The rest of the paper has the following structure. In section 2, we describe the research background. The proposed link prediction algorithm is presented in Section 3. The environmental simulation and performance metric are described in Section 4. Section 5 presents the research results and discussions and Section 6 includes a summary of our research and future work in line with the conclusions and recommendations. 


\section{BACKGROUND AND RELATED WORK}

\subsection{Ad-Hoc On-Demand Distance Vector Routing Protocol (AODV)}

AODV is a reactive routing protocol developed by Perkins and Royer [11] and its multi-hop routing and discovery protocol are effective upon request. The AODV protocol includes the benefits of the DSDV and DSR protocols [11]. In the route discovery process, the source node broadcasts a route request (RREQ) packet across the MANET nodes and sets the time to wait. The RREQ packet contains routing information including the IP address of the originator, the broadcast ID, and the target sequence number.

To preserve the reverse route to a source node, any intermediate node receiving the RREQ packet conducts two operations. The intermediate node first checks whether the RREQ packet was sent before with the same IP and broadcast ID source address and then decides whether the RREQ packet should be refused or accepted. The intermediate node must also test the destination sequence number stored in its routing table if the RREQ packet is accepted. The intermediate node uni-casts the Path Reply (RREP) packet in the source node if the sequence number reaches or matches the sequence number registered in the RREQ packet. If no intermediate node has a fresh enough path to the destination node, the RREQ packet may begin to traverse until the destination node is reached

Figure 1 indicates the source node (S) that sends RREQ packets to its adjacent nodes over the network until the RREQ packet reaches the destination node (D). The destination (D), which replies to the source node using an RREP, is shown in Figure 2.

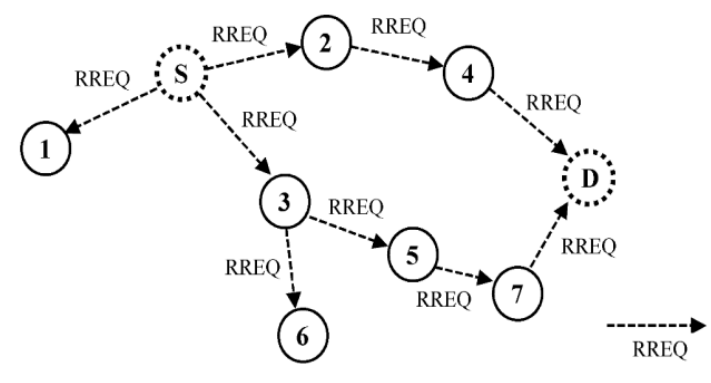

Figure 1. AODV broadcasts RREQ packet

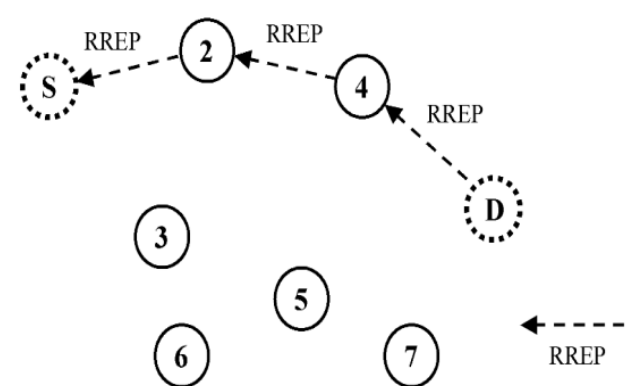

Figure 2. AODV replies RREP packet

Often in AODV, each network node sent a "Hello" packet regularly to maintain its one-hop neighbor routing table. A "Hello" packet is used to evaluate whether the adjacent link is still active. The node sends "Hello" packet with a time interval called "Hello"-interval to its neighbor node to mark broken links between the nodes. Each node sends "Hello" packets to its surroundings and receives its acknowledgment. If a node sends "Hello" packets to a neighbor twice and has not received a message of acknowledgment for it, then the node initiates the broken connection process. If the connection node is near the destination node (i.e., the hop number to the destination node is less than that of the hops to the source node), a new route, known as Local Repair, is necessary to reach the destination node.

Figure 3 shows, for example, the local repair process when the connection between node 4 and node D has been disrupted. In this case, node 4 propagates a Route Error Packet (RERR), which contains addresses of the unreachable destination, to the source node. The routes which have an 
unattainable destination node that is the RERR propagation node, are no longer visible and propagate the RERR once again. When the source nodes collect the RERR and the route rediscovered, the path to the destination node is equally invalid. [12].

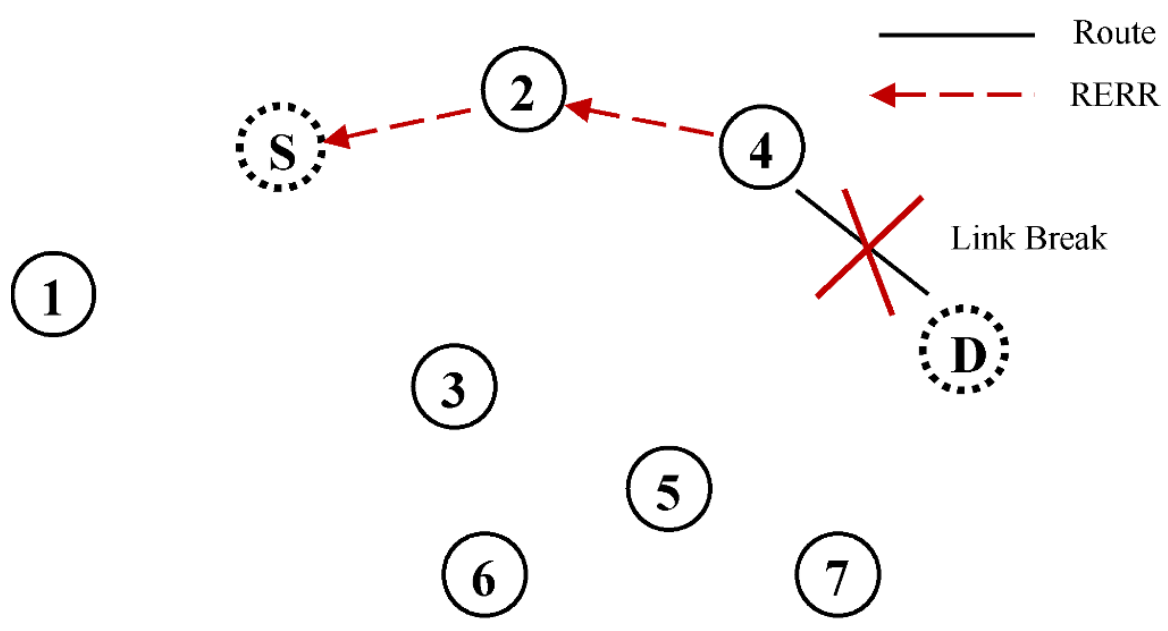

Figure 3. AODV RERR packet

\subsection{Related work}

In the literature of MANET routing protocols, some mobility prediction strategies and methods have been suggested. This research surveys the mobility link break studies that focus on predicting the link breakage between the neighboring nodes.

In [13], the authors proposed two predictive link efficiency techniques that are used during control packet routing. The first technique known as regionally based approximation divides each node's transmission distribution zone into three regions, i.e., internal, central, and outer, on the basis of its signal strength. While nodes in the inner and outer regions are removed from the control packet transmission as their respective signal strength is exceptionally high and low. In the middle area, ties for power packet traffic are only allowed within the region. The second method consists of increasing the region-based estimation, in which nodes are regarded for the selection of routes in the local field and are named segment-based estimates. Based on the current location, the neighboring segment, and the region in which the adjudicator is positioned within the propagation field, the connection-availability ratio of the node for each corresponding path is defined. Such information assists in the flow of control packets inside the outer zone. Simulation of control overhead, number of the compliance packet, route length, and number of route errors within the network was conducted via DSR and AODV protocols. The results show that familiarity with the link status during routing allows identifying more trusted routes with less overhead control, thereby increasing the overall network efficiency.

In [14], an enhanced AODV-based Randomized Link Repair (RLRAODV) routing protocol is suggested that uses multiple route response packets for data transmission across alternative paths during link loss. The suggested protocol reduced the overhead incurred by network congestion due to the exchange of packets for path requests. The analyzes of the simulation show that RLRAODV performs better than AODV performance parameters such as delay, packet arrival, and Packet Loss.

In [15], the authors examine a new mobility prediction approach using movement history and existing genetic algorithms to enhance MANET routing algorithms. The suggested lightweight 
genetic algorithm uses the weighted roulette wheel algorithm to execute outlier elimination, based on heuristics and parent selection [16]. An adjacent node-to-node matrix is produced after genetic operations using force-directed graphs and measuring of vectors, which determine the expected position of each node. The approach proposes a new mechanism to mobility prediction, based exclusively on genetic algorithms and not on probabilistic methods.

In [17], the authors suggested a signal strength-based connection quality prediction approach that should be used in AODV routing. The method is called AODV Link Prediction (AODVLP). The AODVLP nodes foresee the disruption duration and comment on the route to the other node connection breaks. Also, the rebuilding of the existing road or finding the new route is carried out far faster than the failure on that basis. This reduces losses and delays in end-to-end data packets. The suggested method is compared to AODV without predicting linkages. The results show a substantial decrease in packet drops and typical end-to-end delays. The data packet delivery ratio of AODV and the link estimation are also being improved.

In [18], the authors present the impacts of location information on the efficiency of greedy stateless routing protocols, resulting from network parameters like beacon packet interval time and node movement speed, on the impact of the location inaccuracy. The authors introduced a fuzzy logic-dynamic beaconing strategy to improve the efficiencies of a nearby node list by increasing the period between beacon packets to deal with inaccuracies in the location information of a neighborhood node list. Using a fuzzy logic control mechanism, optimization is based on the association between node movement speed, the number of neighboring nodes, and time of the beacon packet interval. The simulation results showed the effectiveness of the fugitive approach in improving the overall efficiency of greedy stateless perimeter routing for beacon packet overhead control end-to-end delay non-optimal hop and false node location influence.

In [19], the authors developed a failure recovery approach for multicast network routing. The approach built an independent route path to overcome a single link or single node breaks down. This proposed approach has the advantages which enable it to build a tree of multicast protection that provides immediate recovery of failure for any individual node failures.

In [20], the researchers have proposed an enhanced Local Preventative Repair Mechanism (PLRM) for the Wireless Ad-hoc Sensor Network (WASN) AODV routing protocol. By monitoring the quality of the links and other performance metrics such as traffic load and remaining energy, PLRM prevents link breakage. The proposed mechanism reduces route length, overhead control and packet delay by documenting certain specific measures using the local repair tool. PLRM shows better efficiency by simulations in terms of packet delay, overhead control and packet delivery ratio in WASN compared with other improved AODV route repairing schemes.

Han et al., in [21], proposed a novel Connection Based Routing Protocol (LBRP) for MANET was suggested. Contrary to current routing protocols, the current LBRP perceived an unpredictable topology change and several connection failures. The distance between the two neighboring nodes is roughly estimated. The main benefit of the proposed protocol is that over a short period it is possible to predict reliable availability of links. LBRP acts as a foundation for a robust routing protocol contrasted with other routing protocols and can help enhance upper-level facilities, reliability, efficiency and quality of service.

\section{Proposed Link Prediction Algorithm}

In a MANET, the collection of wireless devices must perform both the data transmit/receive function and the routine function. A MANET is self-organized network because wireless devices have limited transmission range; a transfer requires the existence of a path, which will typically 
consist of multiple hops. The network topology continues to change unpredictably because of the mobility of these devices. This means that path discovery and maintenance is an essential function in a MANET.

This section is mainly aimed at giving priority to link repair and/or link detection. To estimate the stability of a route consisting of multiple links, it is necessary to assess which links are likely to become unavailable. A very substantial part of the MANET algorithm is devoted to linking management and prediction [22]. The proposed algorithm was developed to estimate when the signal strength of a link will fall below some given threshold. This is used to imply that the two nodes in question move away. The link prediction algorithm acts as a proactive algorithm in that it warns that source that a new path must be found before the existing path has collapsed. This gives the node of origin the opportunity for path discovery while a current path is still in place. The examination phase will be held on each neighbor through whom three measurements "represent the received three last data packets with signal strength" for power and time will be acquired. Using the values of both measures, we can examine the state of the packet in fixed signal power. If the stack depth "represent buffer queue at source and destination" is higher than 3 degrees, an increment of the packet time and energy will be established to predict the time $t_{p}$ at fixed single power threshold $p=3.65 \times 10-11$ (used as an indication to predict the breakage in the link). However, if the time value obtained from the packet is equal to the critical juncture parameter $t_{s}$ for creating a new path, then calculate $t_{p}$ and call local route repair.

The equations to predict the link failure of signal strength received packets, using the Newton divided difference method less than the total number of observations. Thus, in our case, it is necessary to solve a quadratic equation to obtain $t_{p}$. If we make the following identifications:

$$
\begin{aligned}
& A=\left(p_{2}-p_{1}\right) /\left(t_{2}-t_{1}\right) \\
& B=\left(\frac{\left(p_{3}-p_{2}\right)}{\left(t_{3}-t_{2}\right)}-\frac{\left(p_{2}-p_{1}\right)}{\left(t_{2}-t_{1}\right)}\right)
\end{aligned}
$$

where $t_{1}, t_{2}, t_{3}$ is a time when the packet arrives, $p_{1}, p_{2}, p_{3}$ is signal power strength for three data packets received.

Note that we can use the same equations for both the original AODV and the modified AODVLBP. The symbols have a different meaning. In AODVLBP, the values of $p$ and $t$ are the values for the three most recently received packets and $p_{r}$ is the threshold signal power. In ordinary AODV, such history may not exist, and therefore, it may be necessary to synthesize the history by the broadcasting of RREQ to get the data values. In other words, in AODVLBP, all $p$ and $t$ values are in the past, while in AODV, some might be in the future.

Simple algebra shows that the Newtonian approximation can be written as:

$$
B t_{p}^{2}+\left(A-B t_{1}-B t_{2}\right) t_{p}+\left(p_{1}-p_{r}-A t_{1}+t_{1} t_{2} B\right)=0
$$

This has the solution:

$$
t_{p}=\frac{-b+\sqrt{b^{2}-4 a c}}{2 a}
$$


International Journal of Computer Networks \& Communications (IJCNC) Vol.12, No.6, November 2020

where $a=B, b=\left(A-B t_{1} B t_{2}\right)$ and $c=\left(p_{1}-p_{r}-A t_{1}-B t_{1} t_{2}\right)$

We describe $t_{s}$ as the time it takes for the source node to find a new path or to repair an established path. However, the proposed changes to the stated link prediction algorithm for both AODV and AODVLBP are explained in the following steps:

1. For each neighbor

2. On receipt of a packet

3. Push (power, time) for packet onto history packet stack; drop older entries if stack depth $>3$

4. If $\min \left(p_{i}<p\right)$ then predict $t_{p}$

5. Predict $t_{p}$

6. 1

7. Use Newtonian variance to calculate $t_{p}$; choose $N$ so that $t_{s}+3 N \leq t_{p}$

8. If (current-time $\geq t_{s}$ ) then call local route repair

9. If (current-time $\left.\geq t_{s}+N\right)$ then call local route repair

10. If (current-time $\left.\geq t_{s}+2 N\right)$ then call repair of the local route

11. $\}$

12. Local route repair()

13. 1

14. Search for the path to the next node n;

15. If a route in $K$ hops has been discovered (in time)

16. Use the retraining route.

17. Else

18. Find a route towards destination $D$;

19. If ( path is found)

20. $\quad$ I

21. Route the packet in a new route

22. Sends the source a reply seeking the shortest route

23. $\quad\}$

24. $\}$

1. At the source:

2. \{

3. Message received for new route discovery,

4. Find a new route,

5. Direct traffic by the new route.

6. $\}$

where:

stack depth: represent buffer queue at source and destination

$t_{p}$ : time predicts

$t_{s}$ : It requires time to find a new path for a source node

K: routing hops through Time To Live (TTL)

The topology diagram for the proposed link prediction algorithm is shown in Figure 4. 


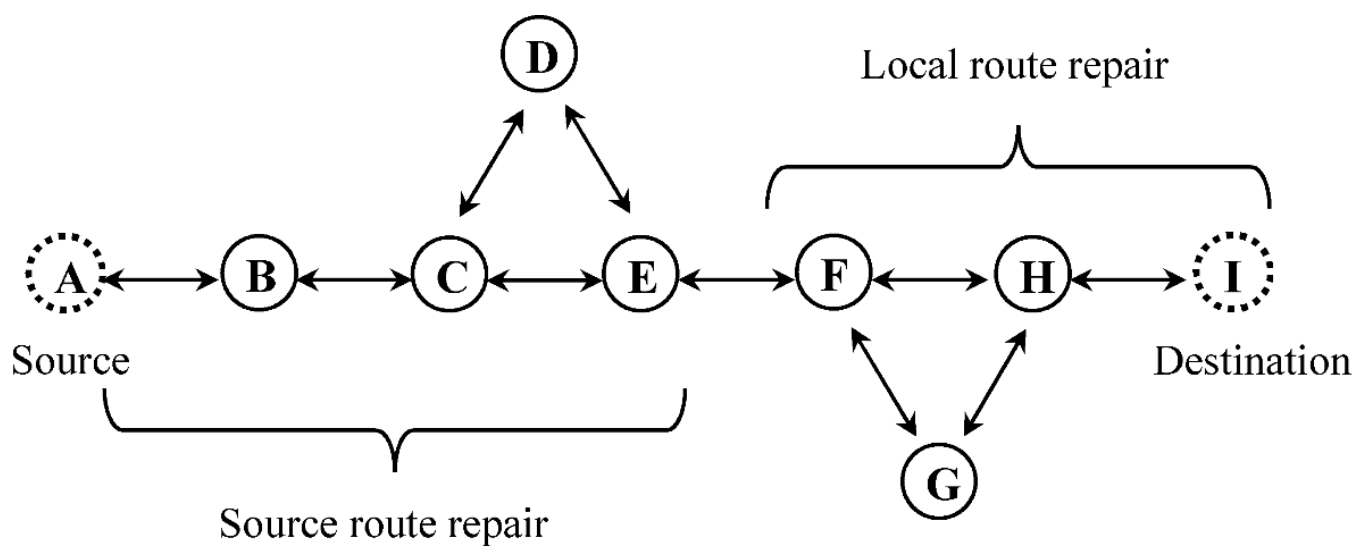

Figure 4. Topology diagram for AODVLBP, local route repair, source repair algorithms

\section{Simulation EnVironment}

The efficiency of the AODVLBP algorithm was tested with Network Simulator version 2.35 (NS-2.35). NS-2.35is a simulation tool that has been shown to be useful for the study of the dynamic nature of networking. NS2.35 may also be used to test wired and wireless network capabilities and protocols (for example, algorithm routing, TCP, UDP). Users will usually specify these protocols on the network and simulate their respective behaviours

During the simulation experiments, we utilised the simulation parameters used in [17]. The mobility model of nodes takes advantage of the random waypoint model. Wireless nodes move freely and arbitrarily in this paradigm of mobility, without boundary restrictions. At the base, the application layer creates traffic at a Constant Bit Rate (CBR). For every node, the transmission range is $250 \mathrm{~m}$. The pause time for the node is set at 0 to allow node motion at all times. Node speeds of $5,10,15,20$, and $25 \mathrm{~m} / \mathrm{s}$ were chosen for the network scenario, and in all situations, the node number is set to 50. The simulation area, eventually, is set to approx. $670 \mathrm{~m}$ in a $670 \mathrm{~m} 2$ area. Table 1 outlines the parameters of the simulation.

Table 1. Simulation parameters

\begin{tabular}{|l|l|l|}
\hline Parameter & Value & Unit \\
\hline No. of Nodes & $10,25,50,75,100$ & node \\
\hline Network Area Size & $670 \times 670$ & $\mathrm{~m}^{2}$ \\
\hline Simulation Time & 300 & $\mathrm{~s}$ \\
\hline Maximum Connections & $5,10,15,20,25$ & count \\
\hline Node Speed & $5,10,15,20,25$ & $\mathrm{~m} / \mathrm{s}$ \\
\hline Type of Traffic & CBR & - \\
\hline Mobility Model & Randomly moving way & - \\
\hline Routing Protocols & AODV, AODVLP, AODVLBP & - \\
\hline Data Packet Size & 512 & bytes \\
\hline
\end{tabular}

This research uses qualitative analysis to gain insights into evaluating the performance metrics of AODVLBP, AODV standard, and AODVLP routing protocols in the three scenarios. NS2.35 is used for simulating results, various simulations with the same parameters have been performed and the average experiential values have been used to minimize the roughness error. The 
performance of three protocols is evaluated in terms of average end-to-end delay, packet delivery ratio, packet overhead ratio, and packet drop-neighbor break.

\subsection{Average End-to-End (E2E) Delay}

The average time delay parameter is the total time needed to pass the data packet successfully via the network from the source to the destination. The average data packet delay for the E2E can be determined using the following formula:

$$
\text { AvgE2Edelay }=\frac{\sum_{i-1}^{n}\left(R_{i}-S_{i}\right)}{n}
$$

where $R i$ is the total received packet, $S i$ is the total sent packet, and $n$ is the number of data packets.

\subsection{Packet Delivery Ratio (PDR)}

The PDR parameter is the total amount of data packets that the targets collect divided by the total number of data packets sent from the source. It is calculation demonstrates how a protocol moves packets successfully from the source to the destination. A high packet distribution ratio indicates positive results that reflect the routing protocol's fulfillment and correctness. The delivery ratio for the packets is determined using the following formula:

$$
P D R=\frac{\text { Spacketsreceivedbydestinations }}{\sum \text { packetssentbysources }} * 100
$$

\subsection{Packet Overhead Ratio (POR)}

The overhead routing ratio statistic relates to a calculation of the total number of routing packets received and data packets sent to the amount of routing packets delivered. This calculation gives an understanding of the required bandwidth to provide data traffic. The overhead routing is calculated using the following formula:

$$
P O R=\frac{\text { Noofroutingpackets }}{\text { Noofroutingpackets }+ \text { Noofdatapacketssent }} * 100
$$

\subsection{Packet Drop-Neighbor Break}

Drop-Neighbor Packet Break is characterized as the number of drops in data packets due to the failure of communication between the source node and the destination during packet transmission $[23,24]$.

\section{RESUltS AND DiscuSSIONS}

\subsection{The Effect of the Number of Nodes}

We used a set of nodes between 10 and 100 to examine the impact of node numbers on the efficiency of AODV, AODVLP, and AODVLBP. Other simulation variables are laid as indicated in Table 1, excluding the number of connections set at 5 and node speed set at $5 \mathrm{~m} / \mathrm{s}$.

The average delay of the last three algorithms is shown in Figure 5. AODVLBP has a shorter delay than with other algorithms. As the number of nodes in AODV, AODVLP, and AODVLBP rises, the time delay for end-to-end decreases. This is because the collision of the packets 
decreases with high node density which contributes to the retransmission of packets. This collision allows the data packet to be forwarded many times to an unattainable neighbor and thus extends the delay for the packet from end to end.

Several nodes with a packet delivery ratio are compared in Figure 6. For the packet delivery ratio, it can be noted that AODVLBP outperforms AODV and AODVLP at all network sizes and approaches near-perfect performance for large networks. This is a particularly important use case since reliable packet delivery in a large mobile network is a very common scenario. Notice also that AODV seems to have achieved convergence at a value of about 94\%, and AODVLP achieved near to $97 \%$ while AODVLBP is close to $100 \%$. This is a significant advantage for AODVLBP because it shows a measurable difference in the (undesirable) retransmission of dropped packets. AODVLBP drops almost no packets or minimized, while AODV drops small and AODVLP drops lower, but still measurable some packets dropping.

Figure 7 shows a comparison result for the control overhead packet for several nodes. The result indicates that all algorithms behave poorly. Control packet overhead is significant, even in small networks, and grows linearly to nearly $90 \%$ for AODV, and approximately $80 \%$ for AODVLP. AODVLBP does better than the original AODV and AODVLP in all cases, and even shows a performance enhancement to high networks. This illustrates the paramount property that AODVLBP is stable concerning network size. The control of packet overhead is a difficult problem; even though AODVLBP has shown distinct improvement, the overhead is still massive but less than other algorithms. Control packets overhead greatly outnumber the actual data packets. This is an active area of research, and further analysis is necessary to achieve additional results.

Figure 8 shows a significantly lower number of packet drop-neighbor breakings for AODVLBP, excluding the far left part of the diagram and a limited (10) number of nodes. One can infer that for a little number of nodes the efficiency gained by the link prediction algorithm is not needed since the total length of any path will be slight, and thus, will be discovered quickly. It is also imperative to observe that for large network size (100 nodes) the packet drop rate is almost near zero. This is a robust indication that AODVLBP converges. While the figure shows the AODV and AODVLP also converges, AODVLBP converges to a significantly better value.

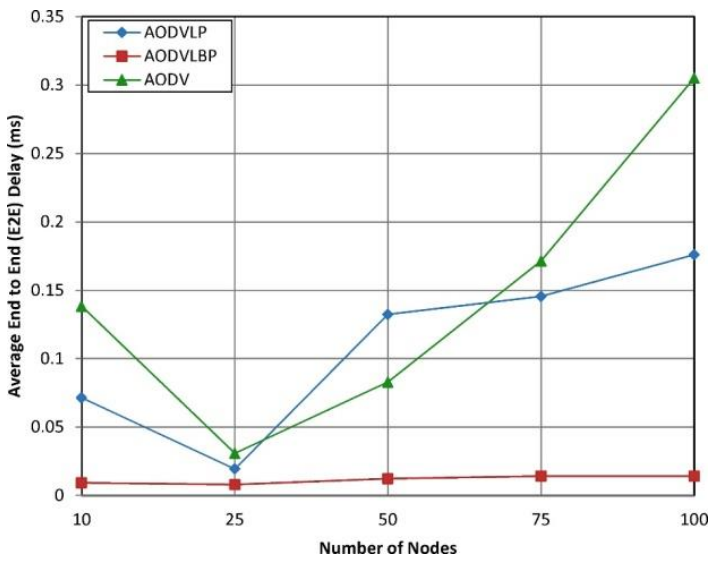

Figure 5. Avg E2E delay against the number of nodes

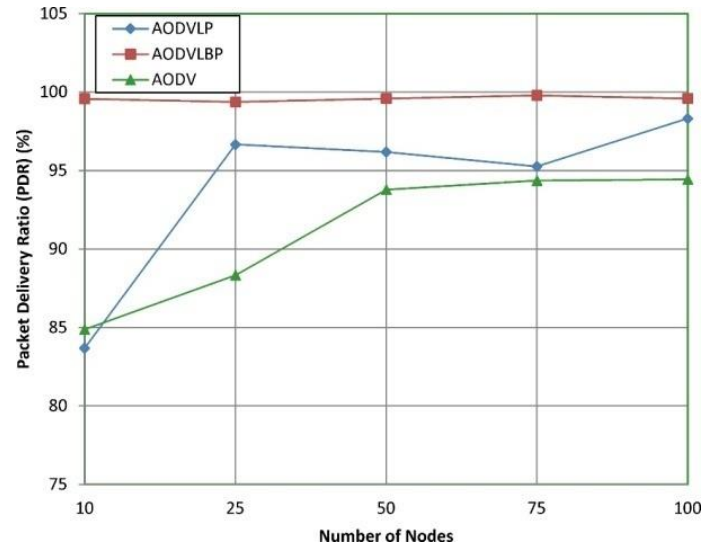

Figure 6. PDR against the number of nodes 
International Journal of Computer Networks \& Communications (IJCNC) Vol.12, No.6, November 2020

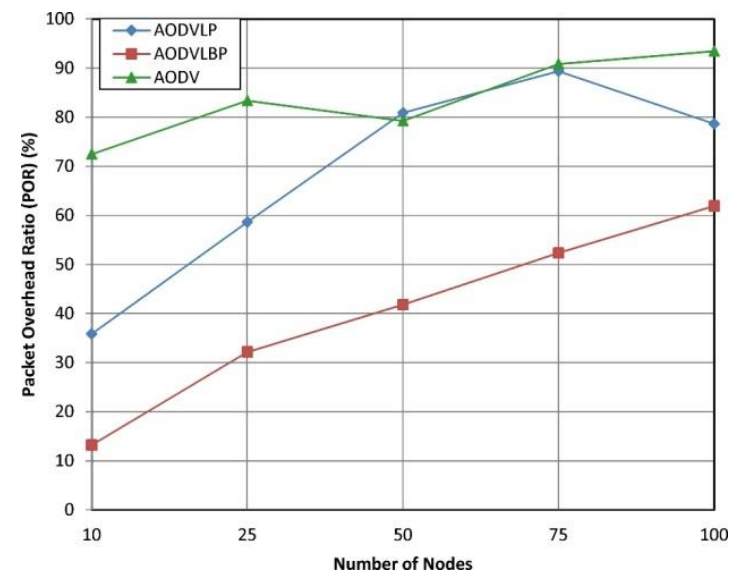

Figure 7. POR against the number of nodes

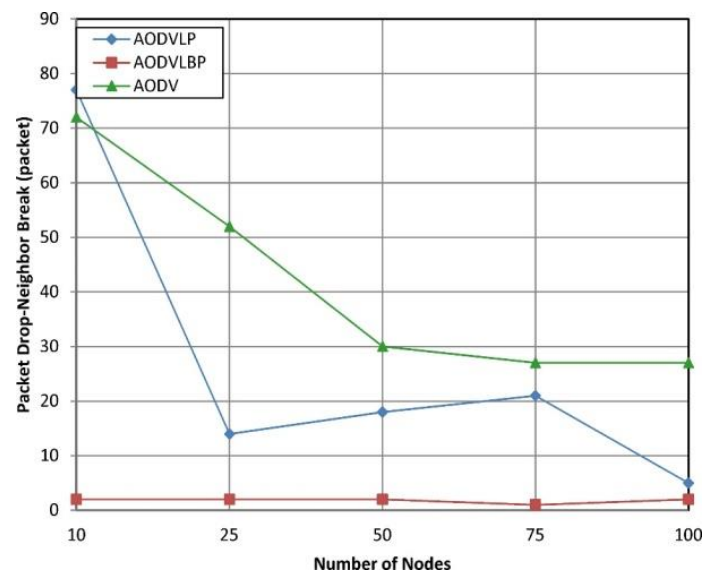

Figure 8. Packet Drop-Neighbor Break against the number of nodes

\subsection{The effect of the number of connections}

The number of connections varies from 5 to 25 to test the effect of the connection number on AODV, AODVLP and AODVLBP performance. As shown in Table 1, all simulation variables are set aside except the number of node sets at 10 nodes and node velocity sets at $5 \mathrm{~m} / \mathrm{s}$.

The typical end-to-end interval in Figure 9 is almost functionally identical to Figure 5. Our algorithm obtained lower percentages in performance metrics of end to end delay. Figure 9 indicates that the total latency for end-to-end also reduces with increasing numbers of connections. The increase in the number of network connections allows further data packets to be re-routed to different paths and thus vulnerable to the breakage of the link.

Figure 10 shows that the proposed AODVLBP algorithm has appreciably outpaced both AODVLP and AODV, as evidenced in the data. This is due to the proposed algorithm selects the reliable node and lesser controls packet leading to the transfer of more data packets.

Figure 11 shows a definite advantage to AODVLBP when measuring control packet overhead versus maximum connections for traffic control. This leads us to infer that the AODVLBP algorithm does a better performance at predicting link breakage and fixing it in high traffic load situations than conventional AODV and AODVLP. The standard anomaly for slight network connectivity appears again in this figure since we can see a substantially higher overhead rate at a small connection size. The other end of the graph shows this too, where AODVLBP is considerably improved.

In Figure 12, the packet drop-neighbor break for AODVLBP is shown as having better performance. As the number of connections increases, there is a growing risk that certain links will be interrupted and a significant number of link failures will occur. Either the algorithm does not address that situation well, or further study is needed for the best alternative to be determined.Moderately well-connected neighborhoods will have a small packet drop-neighbor break. As the number of connections grows, the packet drop rate increases steeply for both algorithms AODV and AODVLP. The inference here seems to be that the number of links (about 20 connections) that can be held is a certain upper limit. Once again, this presents a difficult problem to solve. The number of connections is a global parameter, so local adjustments to the routing algorithms will likely introduce only small (or zero) improvements. 
International Journal of Computer Networks \& Communications (IJCNC) Vol.12, No.6, November 2020

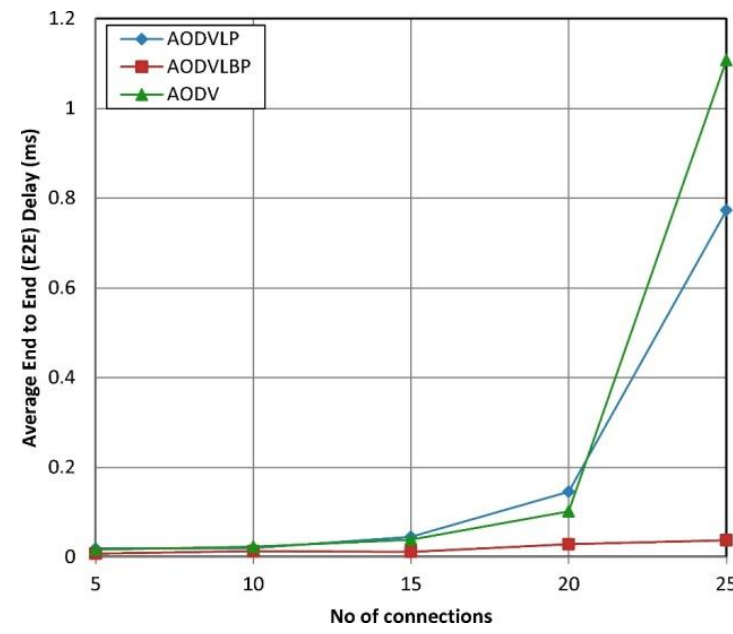

Figure 9. Avg E2E delay against the number of connections

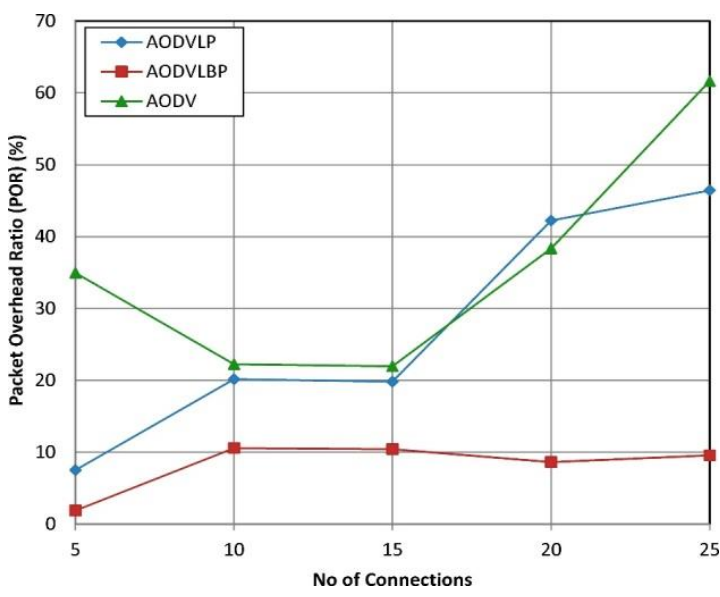

Figure 11. POR against the number of connections

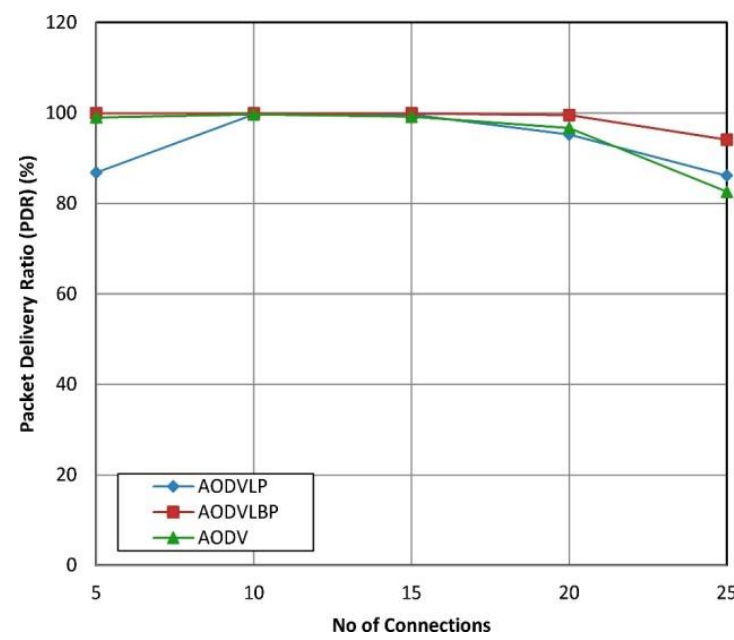

Figure 10. PDR against the number of connections

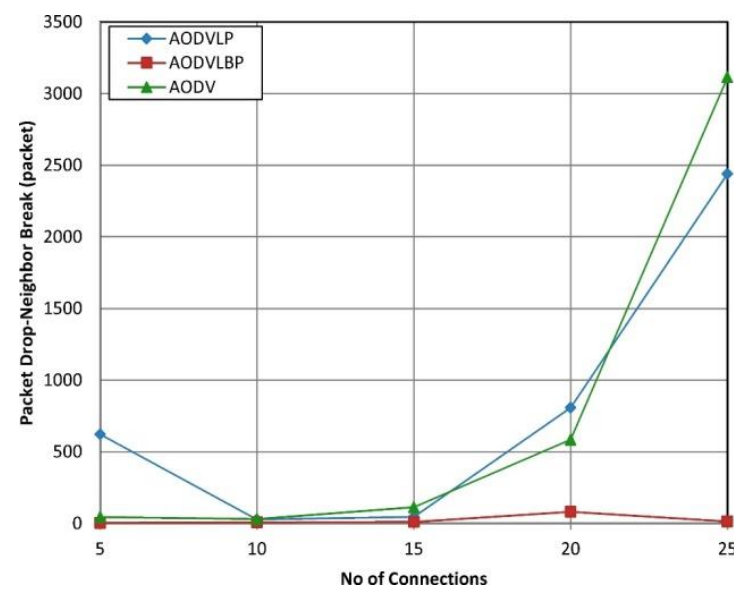

Figure 12. Packet Drop-Neighbor Break against the number of connections

\subsection{The Effects of Node Speed}

The node speed ranges from $5-25 \mathrm{~m} / \mathrm{s}$ to investigate the effect of node speed on AODV, AODVLP, and AODVLBP performance. Other simulation variables are set aside as shown in Table 1 except the number of nodes sets to 10 nodes and the number of connections is set to 5 connections.

The average delay for the three algorithms is shown in Figure 13. AODV has a high end-to-end delay relative to AODVLP at low node speed. At the high node speed, AODVLP starts to perform worse than AODV. In general, for low and medium mobility, both AODV and AODVLP behave in approximately the same way. However, at a node speed of roughly $20 \mathrm{~m} / \mathrm{s}$, the AODVLP algorithms begin to show worsening performance that increases sharply where the node speed increases. AODVLBP starts with better performance and keeps slightly change throughout the changing in the node speed.

Figure 14 shows that all algorithms do well on the packet delivery ratio, but AODVLBP does slightly better when the node speed is low. This is undoubtedly related to the short path length. 
Since AODVLBP will discover a path more quickly, it will find a small path very rapidly and thus is expected to do well in a quiet movable node. When the node speed increases, the performance of the AODV and AODVLP algorithms is almost the same. This is probably since the packet delivery ratio is only very weakly correlated to node speed.

As shown in Figure 15, the control packet overhead ratio is very high for all three algorithms. AODV and AODVLP results are also similar and therefore cannot be seen, even if many more nodes are used. Also, this is almost certainly because the timing of the RREQ packets is not correlated with the node speed. Even on very mobility networks, the control packet overhead for both protocols AODV and AODVLP are stable at a considerable value. On the contrary, AODVLBP behaves better performance with a slight increase through node speed increment.

Figure 16 shows packet drop-neighbor breaks for the three algorithms. The performance of AODVLBP is significantly better than other algorithms AODV and AODVLP for increasing node speed. This again reflects the tradeoff between doing early RREQs versus a broadcast. Naturally, the performance of a broadcast-based algorithm will worsen as the node speed grows.

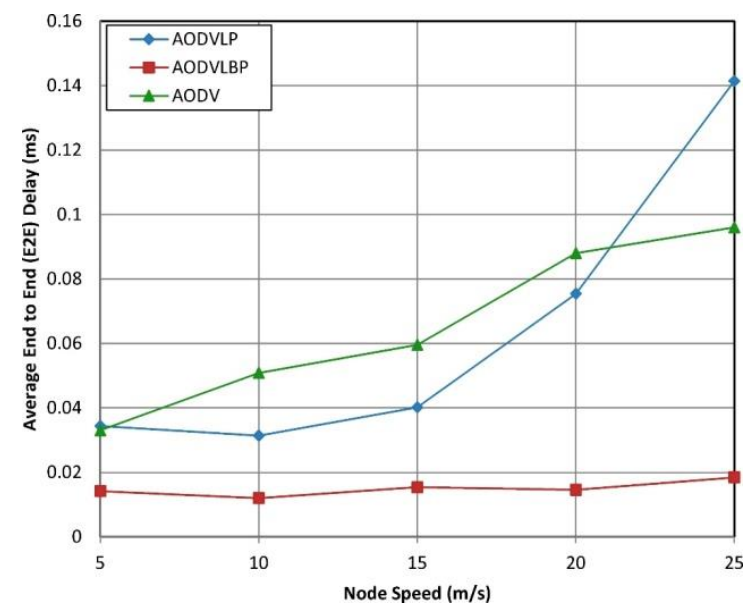

Figure 13. Avg E2E delay against node speed

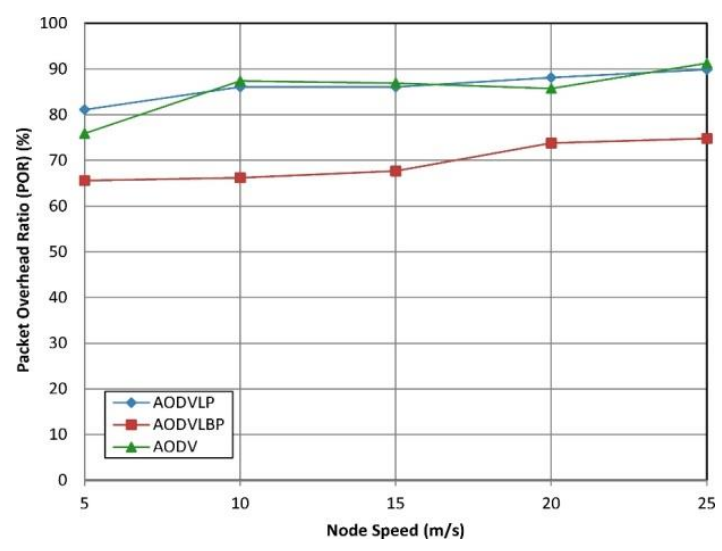

Figure 15. POR against node speed

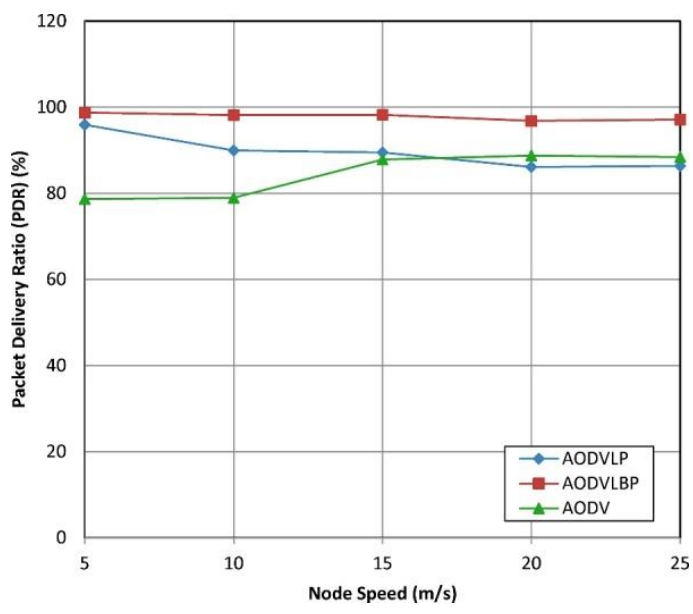

Figure 14. PDR against node speed

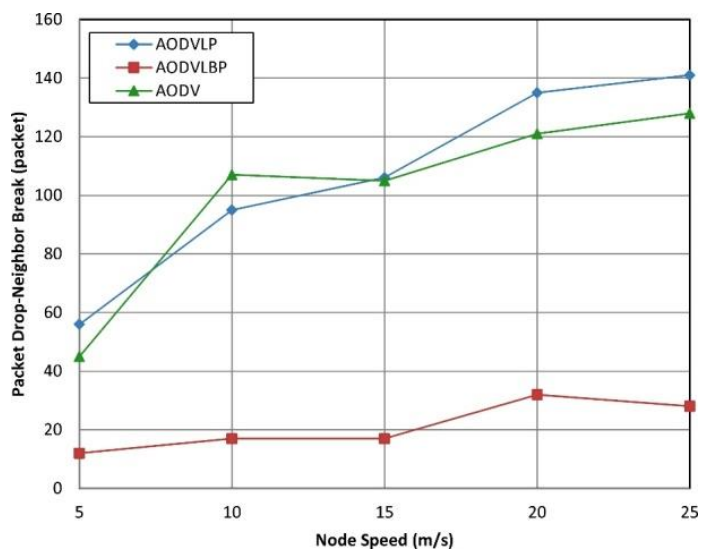

Figure 16. Packet Drop-Neighbor Break against node speed 


\section{CONClusion AND Future WORK}

In this research, a prediction algorithm was suggested for links to improve the prediction of a connection failure in the AODV routing protocol. The foremost goal of this algorithm is to estimate when the signal strength of a link will fall below some given threshold. This is used as an indication that the two nodes in question are moving away from one another. The link prediction algorithm acts as a proactive algorithm in that it cautions that the origin node that a new path must be found before the existing path has collapsed. This gives the source node the opportunity for path discovery while a current path is still in place.

The results of the performance evaluation showed that AODVLBP offered better performance than the original AODV and AODVLP algorithms. Therefore, it can be said that AODVLBP is in many ways a better MANET routing algorithm, which can be effectively used to replace the current AODVLP. The simulation results show AODVLBP's success in improving the overall efficiency of its network in terms of average delay end-to-end, package delivery ratio overhead ratios, and packet drop-neighbor breakage.

Despite the advantages of the proposed link break prediction AODVLBP, some limitations can be avoided in future works. The number of nodes used was limited to 100 nodes only in which examining the performance on larger the number of nodes may slightly lead to different measures. Furthermore, this study was limited to the size of the network which could help distribute the nodes in the given small area.

We plan to extend our work in the future and compare our algorithm with other prediction techniques. In order to achieve better performance, future studies could also consider combining the proposed AODVLBP with other established routing algorithms. In addition, the configurations of power optimization can be used to apply a further improvement to the performance of the algorithm and network.

\section{CONFLICTS OF INTEREST}

The authors declare no conflict of interest.

\section{ACKNOWLEDGMENTS}

This research was funded by the Deanship of Scientific Research at Princess Nourah bint Abdulrahman University through the Fast-track Research Funding Program.

\section{REFERENCES}

[1] C. Reddy, "Node activity based trust and reputation estimation approach for secure and QoS routing in MANET," International Journal of Electrical \& Computer Engineering (2088-8708), vol. 9, 2019.

[2] T. Kunz and S. Ravi, Ad-Hoc, Mobile, and Wireless Networks: 5th International Conference, ADHOC-NOW 2006, Ottawa, Canada, August 17-19, 2006 Proceedings vol. 4104: Springer Science \& Business Media, 2006.

[3] M. Das, B. Sahu, and U. Bhanja, "Mobility and its Effect on the Performance of MANET," in 2015 IEEE Power, Communication and Information Technology Conference (PCITC), 2015, pp. 871-877.

[4] M. B. Talawar and D. Ashoka, "Link Failure Detection in MANET: A Survey," in Modern Approaches in Machine Learning and Cognitive Science: A Walkthrough, ed: Springer, 2020, pp. 169-182.

[5] D. K. Panda and R. K. Dash, "Reliability Evaluation and Analysis of Mobile Ad Hoc Networks," International Journal of Electrical \& Computer Engineering (2088-8708), vol. 7, 2017. 
[6] K. S. Patel and J. Shah, "Study the effect of packet drop attack in AODV routing and MANET and detection of such node in MANET," in Proceedings of International Conference on ICT for Sustainable Development, 2016, pp. 135-142.

[7] C. Dhakad and A. S. Bisen, "Efficient route selection by using link failure factor in MANET," in 2016 International Conference on Electrical, Electronics, and Optimization Techniques (ICEEOT), 2016, pp. 3740-3743.

[8] Y. Bai, Y. Mai, and N. Wang, "Performance comparison and evaluation of the proactive and reactive routing protocols for MANETs," in 2017 Wireless Telecommunications Symposium (WTS), 2017, pp. $1-5$.

[9] A. K. Gupta, H. Sadawarti, and A. K. Verma, "Performance analysis of AODV, DSR \& TORA routing protocols," IACSIT international journal of Engineering and Technology, vol. 2, pp. 226-231, 2010.

[10] M. K. Gulati and K. Kumar, "Performance comparison of mobile Ad Hoc network routing protocols," International Journal of Computer Networks \& Communications, vol. 6, p. 127, 2014.

[11] C. Perkins, E. Belding-Royer, and S. Das, "Ad hoc on-demand distance vector (AODV) routing," 2070-1721, 2003.

[12] A. Khosrozadeh, A. Akbari, M. Bagheri, and N. Beikmahdavi, "A new algorithm AODV routing protocol in mobile ADHOC networks," International Journal of Latest Trends in Computing (IJLTC), vol. 2, pp. 457-464, 2011.

[13] S. R. Malwe, N. Taneja, and G. Biswas, "Enhancement of DSR and AODV Protocols Using Link Availability Prediction," Wireless Personal Communications, vol. 97, pp. 4451-4466, 2017.

[14] S. Rani and T. C. Aseri, "Randomized Link Repair Reactive Routing Protocol for Vehicular Ad Hoc Network," International Journal of Sensors Wireless Communications and Control, vol. 9, pp. 64-79, 2019.

[15] R. Suraj, S. Tapaswi, S. Yousef, K. K. Pattanaik, and M. Cole, "Mobility prediction in mobile ad hoc networks using a lightweight genetic algorithm," Wireless Networks, vol. 22, pp. 1797-1806, 2016.

[16] W. Qian, J. Chai, Z. Xu, and Z. Zhang, "Differential evolution algorithm with multiple mutation strategies based on roulette wheel selection," Applied Intelligence, vol. 48, pp. 3612-3629, 2018.

[17] A. Yadav, Y. N. Singh, and R. Singh, "Improving routing performance in AODV with link prediction in mobile adhoc networks," Wireless Personal Communications, vol. 83, pp. 603-618, 2015.

[18] R. Alsaqour, M. Abdelhaq, R. Saeed, M. Uddin, O. Alsukour, M. Al-Hubaishi, et al., "Dynamic packet beaconing for GPSR mobile ad hoc position-based routing protocol using fuzzy logic," Journal of Network and Computer Applications, vol. 47, pp. 32-46, 2015.

[19] A. Sundarrajan and S. Ramasubramanian, "Fast reroute from single link and single node failures for IP multicast," Computer Networks, vol. 82, pp. 20-33, 2015.

[20] Z. Zhang, Z. Li, and J. Chen, "An enhanced AODV route repairing mechanism in wireless ad-hoc sensor network," Communications, vol. 9, pp. 651-657, 2014.

[21] Q. Han, Y. Bai, L. Gong, and W. Wu, "Link availability prediction-based reliable routing for mobile ad hoc networks," Communications, IET, vol. 5, pp. 2291-2300, 2011.

[22] A. Yadav, Y. Singh, and R. Singh, "Improving Routing Performance in AODV with Link Prediction in Mobile Adhoc Networks," Wireless Personal Communications, pp. 1-16, 2015.

[23] R. A. Alsaqour, M. S. Abdelhaq, and O. A. Alsukour, "Effect of network parameters on neighbor wireless link breaks in GPSR protocol and enhancement using mobility prediction model," EURASIP Journal on Wireless Communications and Networking, vol. 2012, pp. 1-15, 2012.

[24] R. Saqour, M. Shanuldin, and M. Ismail, "Prediction schemes to enhance the routing process in geographical GPSR ad hoc protocol," Mobile information systems, vol. 3, pp. 203-220, 2007. 